\title{
A Transient-Enhanced Low-Quiescent Current Low-Dropout Regulator With Buffer Impedance Attenuation
}

\author{
Mohammad Al-Shyoukh, Hoi Lee, Member, IEEE, and Raul Perez, Member, IEEE
}

\begin{abstract}
This paper presents a low-dropout regulator (LDO) for portable applications with an impedance-attenuated buffer for driving the pass device. Dynamically-biased shunt feedback is proposed in the buffer to lower its output resistance such that the pole at the gate of the pass device is pushed to high frequencies without dissipating large quiescent current. By employing the current-buffer compensation, only a single pole is realized within the regulation loop unity-gain bandwidth and over $65^{\circ}$ phase margin is achieved under the full range of the load current in the LDO. The LDO thus achieves stability without using any low-frequency zero. The maximum output-voltage variation can be minimized during load transients even if a small output capacitor is used.

The LDO with the proposed impedance-attenuated buffer has been implemented in a $0.35-\mu \mathrm{m}$ twin-well CMOS process. The proposed LDO dissipates $20-\mu \mathrm{A}$ quiescent current at no-load condition and is able to deliver up to 200-mA load current. With a $1-\mu \mathrm{F}$ output capacitor, the maximum transient output-voltage variation is within $3 \%$ of the output voltage with load step changes of $200 \mathrm{~mA} / 100 \mathrm{~ns}$.
\end{abstract}

Index Terms-Linear regulator, load transient response, lowdropout regulator (LDO), pass device, power management integrated circuits, voltage buffer.

\section{INTRODUCTION}

$\mathbf{P}$ OWER management is essential in all battery-powered portable devices such as cellular phones and PDAs in order to reduce the standby power and prolong the battery runtime. Low-dropout regulators (LDOs) are one of the most critical power management modules, as they can provide regulated low-noise and precision supply voltages for noise-sensitive analog blocks. With the widespread proliferation of modern portable devices, ever more stringent performance requirements of the LDO are needed. First, low dropout voltage across the pass device of the LDO is required provide high power efficiency. In addition, the increased level of integration in portable devices not only demands the LDO to deliver high load current, but also requires the no-load quiescent current of the LDO to be minimized for improving the current efficiency [1]. Good load transient response with small output-voltage variation including overshoots and undershoots upon load switching is critical to

Manuscript received November 10, 2006; revised April 12, 2007. This work was supported by Texas Instruments Inc.

M. Al-Shyoukh is with Texas Instruments Inc., Dallas, TX 75243 USA, and also with the Department of Electrical Engineering, University of Texas at Dallas, Richardson, TX 75083-0688 USA (e-mail: mshyoukh@ti.com).

H. Lee is with the Department of Electrical Engineering, University of Texas at Dallas, Richardson, TX 75083-0688 USA (e-mail: hoilee@utdallas.edu).

R. Perez is with Fyrestorm Inc., Sunnyvale, CA 94085 USA.

Digital Object Identifier 10.1109/JSSC.2007.900281 prevent an accidental turn off or resetting of the portable device. These four major performance requirements of the LDO, including low dropout voltage, high output current, low no-load quiescent current, and small output transient undershoots and overshoots are, however, difficult to achieve simultaneously.

In LDO design, the ability to source high load current while achieving low dropout voltage requires the use of a large size pMOS transistor as the pass device [1]. In addition to the low-frequency dominant pole generated by the output capacitor, the large gate capacitance of the pMOS pass device creates another low-frequency non-dominant pole within the unity-gain frequency of the regulation loop, thereby degrading stability. Different approaches have been reported to address this issue [1]-[5]. In [1], an emitter-follower has been adopted as a voltage buffer to drive the pMOS pass device. The low output resistance of the emitter-follower allows the pole at the gate of the pass device to be pushed beyond the unity-gain frequency of the LDO regulation loop. The reported LDO dissipates low quiescent current, while sourcing the maximum load current of $50 \mathrm{~mA}$ [1]. However, if the LDO is required source a larger load current (e.g., $100 \mathrm{~mA}$ or more), a much larger pass device with larger gate capacitance is needed. The current dissipation of the emitter-follower thus needs to be greatly increased to further lower its output resistance at the gate of the pass device for maintaining loop stability. Instead of dissipating large quiescent current in the voltage buffer to achieve loop stability, the approach of creating a low-frequency left-half-plane (LHP) zero has been widely employed, which provides positive phase shift to compensate for the negative phase shift due to the low-frequency non-dominant pole [2]-[5]. The low-frequency LHP zero can be generated by either adding a resistor in series with the output capacitor (or the intrinsic equivalent series resistor (ESR) of the output capacitor), namely ESR zero [2]-[4], or relying on frequency compensation through a voltage-controlled current source [5]. However, the exact pole-zero cancellation within the unity-gain frequency of the LDO regulation loop is difficult to achieve under the full range of the load current. The incomplete pole-zero cancellation may lead to instability of the LDO in the worst condition. Small pole-zero frequency mismatch within the unity-gain frequency can degrade the quasi-linear transient settling behavior [6], [7] of the LDO upon load switching. Even worse, if the ESR zero approach is adopted, the resistor leads to large output overshoots and undershoots during massive load-current step changes especially when a low-value output capacitor $C_{L}$ of micro-farad range is used. 


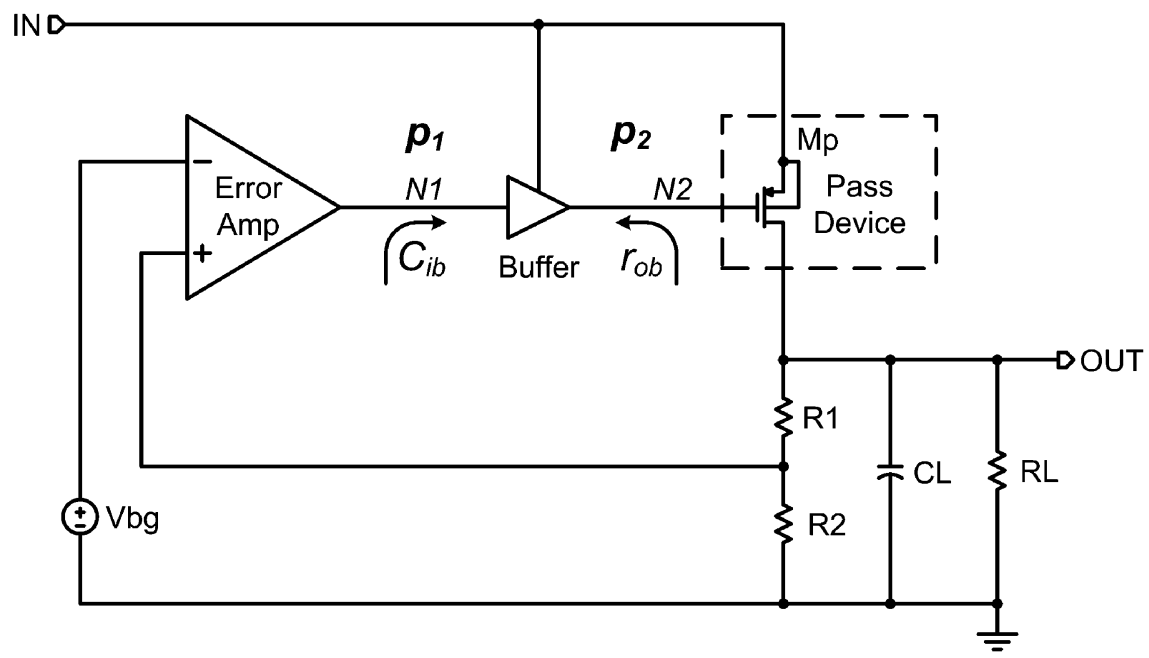

Fig. 1. Typical structure of a low-dropout regulator with an intermediate buffer stage.

In order to further improve LDO performances, buffer impedance attenuation technique (BIA) is first proposed to realize an intermediate stage for driving the pMOS pass device. The proposed BIA technique greatly reduces the output resistance of the buffer through dynamically-biased shunt feedback. As a result, the pole at the gate of the pass device is pushed far beyond the unity-gain frequency of the LDO regulation loop under the entire load current range even if a huge pass device is used for achieving low dropout voltage and sourcing high load current. The BIA technique thus allows the LDO to dissipate low quiescent current. By employing current-buffer compensation in the LDO, only a single pole is realized within the unity-gain frequency and a good phase margin is achieved for the entire load current range with a small compensation capacitor. The LDO thus achieves stability without using any low-frequency zero. Moreover, the LDO can have good transient settling behavior and small output-voltage variation even if a small output capacitor is used.

This paper is organized as follows. Section II presents the operational principle of the proposed BIA technique, while different design considerations including the stability analysis of the LDO using current-buffer compensation and details of LDO circuit implementation are discussed in Section III. Finally, Sections IV and V provide the measurement results of the proposed LDO and the conclusions, respectively.

\section{Proposed ImPedance-Attenuated BufFER}

Fig. 1 shows a typical structure of a low-dropout regulator, which consists of an error amplifier comparing a scaled-down output signal to a bandgap voltage $V_{b g}$, a pMOS pass transistor $M_{p}$, and an intermediate buffer stage driving $M_{p}$. There are three poles in the LDO structure located at the output of the error amplifier $\left(N_{1}\right)$, the output of the buffer $\left(N_{2}\right)$, and the output of the LDO $\left(V_{\text {out }}\right)$. In particular, these poles are given by

$$
\begin{aligned}
p_{1 \mid N_{1}} & =1 /\left(r_{o 1} C_{1}\right) \\
p_{2 \mid N_{2}} & =1 /\left(r_{o b} C_{p}\right) \\
p_{o \mid V_{\text {out }}} & =1 /\left(R_{o e q} C_{L}\right)
\end{aligned}
$$

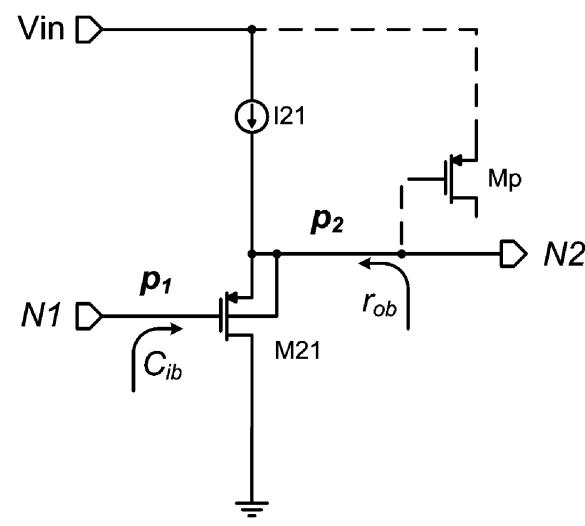

Fig. 2. Source-follower implementation of the intermediate buffer stage.

where $r_{o 1}$ is the output resistance of the error amplifier, $C_{1}$ is the equivalent capacitance at $N_{1}$ which is dominated by the input capacitance of the buffer $C_{i b}, r_{o b}$ is the output resistance of the buffer, $C_{p}$ is the input capacitance of $M_{p}$, and $R_{o e q}$ is the equivalent resistance seen at the output of the LDO. Ideally, both $C_{i b}$ and $r_{o b}$ should be very small in order to achieve single-pole loop response by locating both $p_{1}$ and $p_{2}$ at frequencies much higher than the unity-gain frequency of the regulation loop.

In order to construct the required intermediate buffer stage, a simple pMOS source-follower is first considered to implement the buffer and its structure is shown in Fig. 2. The pMOS source-follower can provide near complete shutdown of the pass device under light-load conditions. Since the output resistance $r_{o b}$ of the source-follower is given by $1 / g_{m 21}$, it is necessary to increase $g_{m 21}$ in order to decrease the value of $r_{o b}$ and allow $p_{2}$ to be located at frequencies much higher than the unity-gain frequency of the LDO regulation loop. Transconductance $g_{m 21}$ can only be increased either through using a larger $W / L$ ratio of transistor $M_{21}$, or through increasing the DC biasing current $I_{21}$ through $M_{21}$, or both. Increasing $I_{21}$ would, however, increase the total quiescent current of the LDO, thereby degrading the current efficiency of the LDO. Using a larger $W / L$ ratio of $M_{21}$ would increase the input capacitance $C_{i b}$ of the buffer, which in turn pushes $p_{1}$ to a lower frequency and the stability can be 


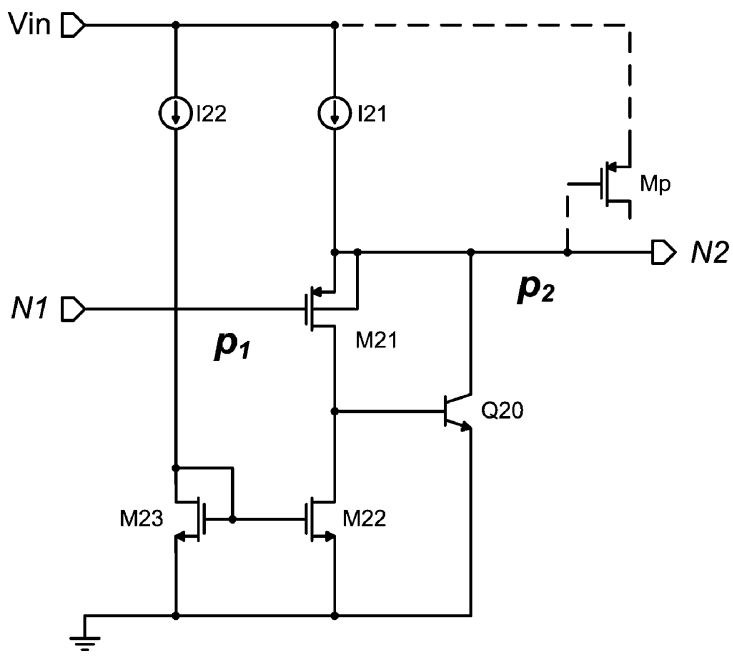

(a)

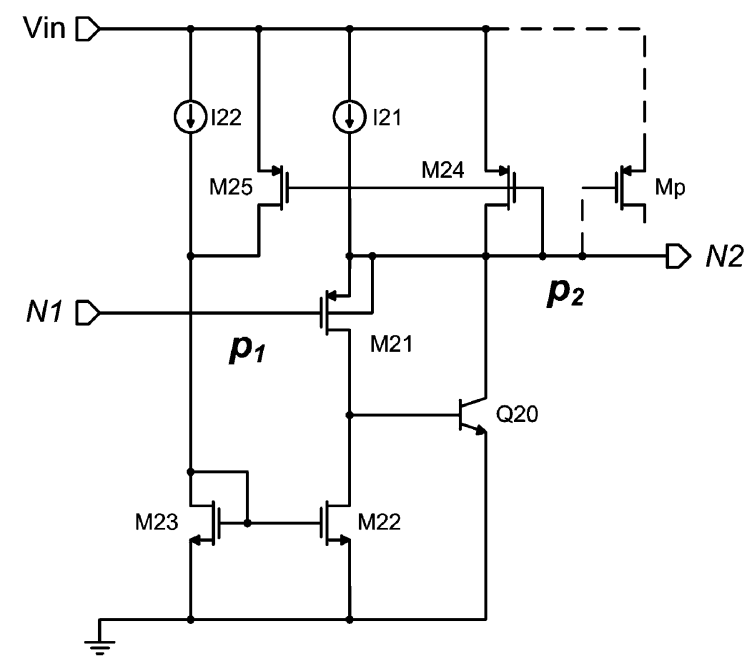

(b)

Fig. 3. (a) Source-follower with shunt feedback and (b) proposed buffer with dynamically-biased shunt feedback for output resistance reduction under different load currents.

poorly affected. A simple pMOS source-follower is, therefore, not a suitable implementation of the intermediate buffer stage in the LDO.

To minimize $W / L$ ratio of $M_{21}$ and the quiescent current required to reach a given $r_{o b}$, the source-follower with negative feedback shown in Fig. 3(a) is used. In particular, the npn transistor $Q_{20}$ is the feedback device connected in parallel to the output of the source-follower $M_{21}$ in order to reduce $r_{o b}$ through shunt feedback. From a qualitative standpoint, when the input voltage at $N_{1}$ is constant and the output voltage increases, the magnitude of the drain current of $M_{21}$ also increases, which in turn increases the base current of $Q_{20}$. As a result, the collector current of $Q_{20}$ increases, reducing the output resistance $r_{o b}$ by increasing the total current that flows into the output node. The output resistance looking into the follower is then given by

$$
r_{o b}=\frac{1}{g_{m 21}(1+\beta)} .
$$

Equation (4) shows that the output resistance of the follower is reduced by the current gain $\beta$ of the shunt feedback device $Q_{20}$. For example, when an npn transistor with $\beta \approx 10$ is used, the value of $r_{o b}$ would be decreased by about 10 times and the frequency of $p_{2}$ at the gate of the pass device is then pushed to a decade higher. As a result, the quiescent current needed through $M_{21}$ is greatly reduced to realize $g_{m 21}$ for a given $r_{o b}$. Similarly, the transistor size of source-follower $M_{21}$ required is also reduced. The input capacitance of the buffer $C_{i b}$ is then decreased, which allows $p_{1}$ given in (1) to be located at a higher frequency without dissipating additional quiescent current.

It should be noted that the shunt feedback device $Q_{20}$ can also be implemented by a nMOS transistor in single-well technologies to achieve a similar reduction in the output resistance [8]. Since inexpensive twin-well technologies with large feature size (0.35 $\mu \mathrm{m}$ and larger) are widely used for integrated power ICs and vertical parasitic npn structures with $\beta>10$ are available in twin-well technologies, the shunt feedback device is implemented by the vertical parasitic npn transistor in our design.
Since the unity-gain frequency of the LDO regulation loop increases with the load current, the output resistance of the buffer should decrease when the load current increases in order to maintain $p_{2}$ far beyond the unity-gain frequency under the entire load current range. Fig. 3(b) shows the proposed structure of the buffer, in which two pMOS transistors $M_{24}$ and $M_{25}$ and the npn transistor $Q_{20}$ realize dynamically-biased shunt feedback to decrease $r_{o b}$ under different load current conditions. The output resistance of the proposed buffer is then given by

$$
r_{o b}=\frac{1}{g_{m 21}(1+\beta)+g_{m 24}}
$$

where $g_{m 24}$ is the transconductance of the diode-connected transistor $M_{24}$. As shown in Fig. 3(b), when the load current flowing through the pass device $M_{p}$ increases, both voltages at $N_{1}$ and $N_{2}$ decrease. The gate-source voltage of $M_{24}$ is increased and hence more current flows through $M_{24}$. This current then mirrors through $M_{25}$ such that the current through the follower device $M_{21}$ dynamically increases with the load current. This boosts the value of $g_{m 21}$, thereby further reducing the output resistance of the buffer according to (5). In addition, the increase in $g_{m 24}$ with the load current can reduce the value of $r_{o b}$. This effect is significant under heavy load current conditions. Moreover, when the load current increases, part of the dynamically-increased current through $M_{21}$ flows into the base of $Q_{20}$ and increases its collector current. The current gain $\beta$ of the vertical parasitic npn transistor slightly increases with the collector current, which also helps on reducing the value of $r_{o b}$ when the load current increases.

The proposed buffer also improves the slewing of the gate drive at $N_{2}$ during load transients. During the transition from no load to full load, the decrease in the voltage at $N_{1}$ causes an increase in the transient current through $M_{21}$, which flows through the base of $Q_{20}$. The collector current of $Q_{20}$ then increases to discharge the large gate capacitance of the pass device $C_{p}$ and the voltage at $N_{2}$ decreases with a faster slewing. It should be noted that $I_{21}$ is designed to be larger than $I_{22}$ such that $Q_{20}$ is always on under different load currents. Similarly, 


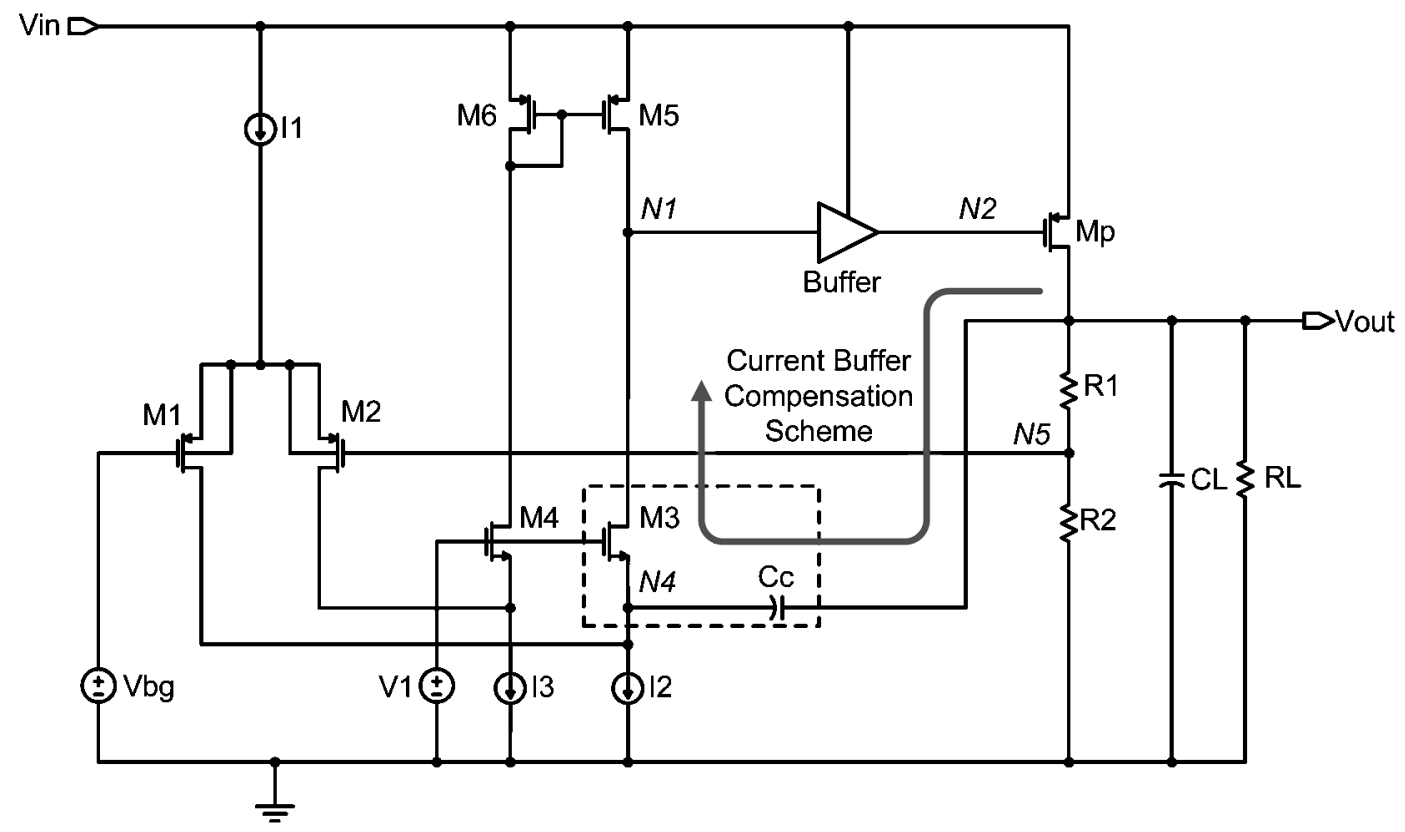

Fig. 4. Structure of the proposed LDO using current-buffer compensation scheme.

when the voltage at $N_{1}$ increases, transistor $M_{24}$ provides extra transient current to charge $C_{p}$ and increase the voltage at $N_{2}$ to follow the voltage change at $N_{1}$ with a faster slewing during the transition from full load to no load.

In short, the proposed buffer with dynamically-biased shunt feedback reduces both the input and output impedance of the buffer by decreasing the values of $C_{i b}$ and $r_{o b}$, respectively. In particular, the reduction in the value of $r_{o b}$ increases with the load current. As a result, $p_{2}$ is located at sufficiently high frequencies under different load currents, while the LDO only dissipates low quiescent current at no-load condition. The benefit of having a smaller $C_{1}$ by using a smaller size of source-follower device in the proposed buffer also improves the stability of the LDO, which will be further clarified in the next section.

\section{Design OF THE PROPOSED LDO}

In the proposed LDO structure shown in Fig. 4, the error amplifier is realized by a single-stage folded-cascode structure with transistors $M_{1}-M_{6}$. As discussed in the previous section, the pole $p_{2}$ at the output of the buffer is pushed to sufficiently high frequencies under different load currents by employing dynamically-biased shunt feedback. The pass device $M_{p}$ realizes a common-source gain stage and constitutes the second gain stage in the LDO. Accordingly, the resulting LDO structure can be viewed as a two-stage amplifier driving a large capacitive load with two poles located at $N_{1}$ and the LDO output. The large capacitive load is due to the use of a micro-farad range off-chip output capacitor $C_{L}$. This LDO structure can be stabilized using current-buffer (or cascode-Miller) frequency compensation, which is a pole-splitting compensation designed for splitting two poles $p_{1}$ at $N_{1}$ and $p_{o}$ at the LDO output in order to achieve stability in the full range of the load current. In twostage amplifier design, the use of the current-buffer compensation scheme allows the amplifier to achieve wider unity-gain frequency and improved stability by removing the right-half-plane zero and enhance PSRR [9]-[15]. In the proposed LDO, current-buffer compensation also removes the right-half-plane zero and improves PSRR. Additionally and more importantly, this compensation allows the LDO to achieve stability over the entire load current range by using only a small compensation capacitor. In Fig. 4, the current-buffer compensation in the LDO is realized by $C_{c}$ and the common-gate transistor $M_{3}$ [15]. The details of the stability using the current-buffer compensation are discussed in the following subsection.

\section{A. Stability Analysis}

The stability of the proposed LDO is studied using the loopgain transfer function $T(s)$ of the regulation loop. Fig. 5 is the small-signal block diagram representation of the circuit in Fig. 4 for analyzing the loop-gain transfer function. Here, $g_{m 1}, g_{m 3}$ and $g_{m p}$ represent the transconductances of input differential stage $M_{1} / M_{2}$, current buffer stage $M_{3}$ and pass device $M_{p}$, respectively. In addition, $B$ is the feedback factor $R_{2} /\left(R_{1}+\right.$ $\left.R_{2}\right)$. As mentioned before, since the output of the buffer $N_{2}$ is a low impedance node and $p_{2}$ is located at a very high frequency under the full range of the load current, the capacitive loading at $N_{2}$ is neglected in the analysis. The loop-gain transfer function is derived based on the following considerations.

1) $C_{L}, C_{c}$ are much larger than $C_{1}$.

2) Transconductance $g_{m p}$ and output resistance $R_{o e q}$ provide the gain of the pass device $A_{p}=g_{m p} R_{o e q}$ and $A_{p}$ varies with the load current $I_{L}$. In particular, $g_{m p}$ $\left(R_{o e q}\right)$ decreases (increases) with the load current and $A_{p}=g_{m p} R_{o e q}$ is inversely proportional to $\sqrt{I_{L}}$.

Based on the above, the loop-gain transfer function of the proposed LDO is given as

$$
\begin{aligned}
T(s) & =\frac{T_{\text {out }}}{T_{\text {in }}}=\frac{-B g_{m 1} g_{m p} r_{o 1} R_{o e q}\left(1+s \frac{C_{c}}{g_{m 3}}\right)}{1+a s+b s^{2}+c s^{3}} \\
a & =R_{o e q}\left(C_{L}+g_{m p} r_{o 1} C_{c}\right)
\end{aligned}
$$




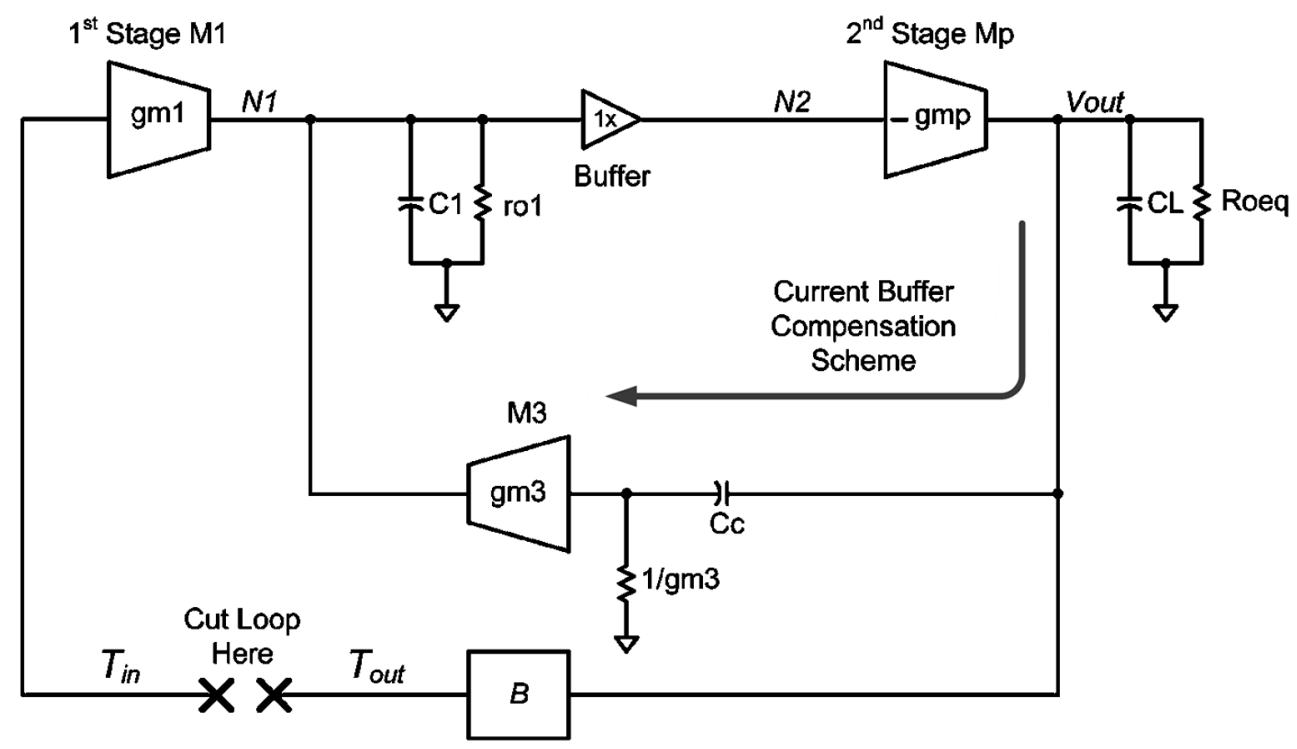

Fig. 5. Small-signal block diagram of the proposed LDO with current-buffer compensation scheme.

$$
\begin{aligned}
& b=C_{1} C_{L} r_{o 1} R_{o e q} \\
& c=\frac{C_{1} C_{c} C_{L} r_{o 1} R_{o e q}}{g_{m 3}} .
\end{aligned}
$$

From (6), the loop gain is negative due to the negative feedback established for output voltage regulation. In addition, the numerator of $T(s)$ indicates that a LHP zero $z_{b}=g_{m 3} / C_{c}$ exists, while the third-order polynomial of the denominator implies that the system has three poles. According to (7)-(9), locations of poles vary under different load currents due to the existence of $R_{o e q}$ and $g_{m p}$. As a result, the loop gain transfer function should be studied for different load current conditions.

When $I_{L}=0$, the current drain from the pass device is $V_{\text {out }} /\left(R_{1}+R_{2}\right)$, which is around 1-3 $\mu \mathrm{A}$. Hence, $g_{m p}$ is at its minimum and $C_{L} R_{o e q} \gg g_{m p} r_{o 1} C_{c} R_{o e q}$ in (7). As a result, $T(s)$ in (6) can be approximated to

$$
\begin{aligned}
& \left.T(s)\right|_{I_{L}=0} \\
& \approx \frac{-B g_{m 1} g_{m p} r_{o 1} R_{o e q}\left(1+s \frac{C_{c}}{g_{m 3}}\right)}{1+s C_{L} R_{o e q}+s^{2} C_{1} C_{L} r_{o 1} R_{o e q}+s^{3} \frac{C_{1} C_{c} C_{L} r_{o 1} R_{o e q}}{g_{m 3}}} \\
& \approx \frac{-B g_{m 1} g_{m p} r_{o 1}\left(1+s \frac{C_{c}}{g_{m 3}}\right)}{\left(1+s C_{L} R_{o e q}\right)\left(1+s C_{1} r_{o 1}\right)\left(1+s \frac{C_{c}}{g_{m 3}}\right)} \\
& =\frac{-B g_{m 1} g_{m p} r_{o 1} R_{o e q}}{\left(1+s C_{L} R_{o e q}\right)\left(1+s C_{1} r_{o 1}\right)} \\
& =\frac{-B g_{m 1} g_{m p} r_{o 1} R_{o e q}}{\left(1+\frac{s}{p_{-3 \mathrm{~dB} \mid I_{L}=0}}\right)\left(\frac{s}{p_{\text {nd } \mid I_{L}=0}}\right)}
\end{aligned}
$$

where $p_{-3 \mathrm{~dB} \mid I_{L}=0}=1 /\left(C_{L} R_{o e q}\right)$ and $p_{\text {nd } \mid I_{L}=0}=1 /\left(C_{1} r_{o 1}\right)$ are dominant and non-dominant poles of $\left.T(s)\right|_{I_{L}=0}$. As indicated in (10), the system is designed to have three separate poles and the third pole is canceled by the LHP zero, resulting in a second-order system. In particular, $p_{-3 \mathrm{~dB} \mid I_{L}=0}=p_{O}$ and $p_{\text {nd } \mid I_{L}=0}=p_{1}$ indicate that no pole splitting occurs during $I_{L}=0$ and both the dominant and non-dominant poles are located at the LDO output and the output of the error amplifier, respectively. With the proposed buffer-impedance attenuation technique, the value of $C_{1}$ can be minimized due to the smaller source-follower device. The small $C_{1}$ allows $p_{\text {nd } \mid I_{L}=0}$ to be much higher than the unity-gain frequency of the loopgain transfer function, which guarantees the proposed LDO to achieve good phase margin under $I_{L}=0$.

When the load current significantly increases, $g_{m p}$ becomes much larger. Hence, $g_{m p} r_{o 1} C_{c} R_{o e q} \gg C_{L} R_{o e q}$ in (7), and $T(s)$ in (6) is approximated to

$$
\begin{aligned}
& \left.T(s)\right|_{I_{L} \gg 0} \\
& \approx \frac{-B g_{m 1} g_{m p} r_{o 1} R_{o e q}\left(1+s \frac{C_{c}}{g_{m 3}}\right)}{1+s g_{m p} C_{c} r_{o 1} R_{o e q}+s^{2} C_{1} C_{L} r_{o 1} R_{o e q}+s^{3} \frac{C_{1} C_{c} C_{L} r_{o 1} R_{o e q}}{g_{m 3}}} \\
& \approx \frac{-B g_{m 1} g_{m p} r_{o 1} R_{o e q}\left(1+s \frac{C_{c}}{g_{m 3}}\right)}{\left(1+s \frac{1}{g_{m p} C_{c} r_{o 1} R_{o e q}}\right)\left(1+s \frac{C_{1} C_{L}}{g_{m p} C_{c}}\right)\left(1+s \frac{C_{c}}{g_{m 3}}\right)} \\
& =\frac{-B g_{m 1} g_{m p} r_{o 1} R_{o e q}}{\left(1+s g_{m p} C_{c} r_{o 1} R_{o e q}\right)\left(1+s \frac{C_{1} C_{L}}{g_{m p} C_{c}}\right)} \\
& =\frac{-B g_{m 1} g_{m p} r_{o 1} R_{o e q}}{\left(1+\frac{s}{p_{-3 \mathrm{~dB} \mid I_{L} \gg 0}}\right)\left(\frac{s}{p_{\mathrm{nd} \mid I_{L} \gg 0}}\right)} .
\end{aligned}
$$

Equation (11) shows that the loop gain transfer function has also two poles under $I_{L} \gg 0$, where $p_{-3 \mathrm{~dB}} \mid I_{L} \gg 0=$ $1 /\left(g_{m p} C_{c} r_{o 1} R_{o e q}\right)$ and $p_{\text {nd } \mid I_{L} \gg 0}=\left(g_{m p} C_{c}\right) /\left(C_{1} C_{L}\right)$ are the dominant and non-dominant poles of $\left.T(s)\right|_{I_{L} \gg 0}$. Under this condition, the maximum unity-gain frequency of the loop-gain transfer function is achieved $\left(\omega_{u, \max }=g_{m 1} / C_{c}\right)$, which is fixed with respect to the load current change. Since $p_{\text {nd } \mid I_{L} \gg 0}$ is always larger than $\omega_{u, \max }$, only a single pole exists within the unity-gain frequency of the regulation loop. In addition, $p_{\text {nd } \mid I_{L} \gg 0}$ increases with the load current, which implies that the phase margin and stability of the LDO improve with the load current. 

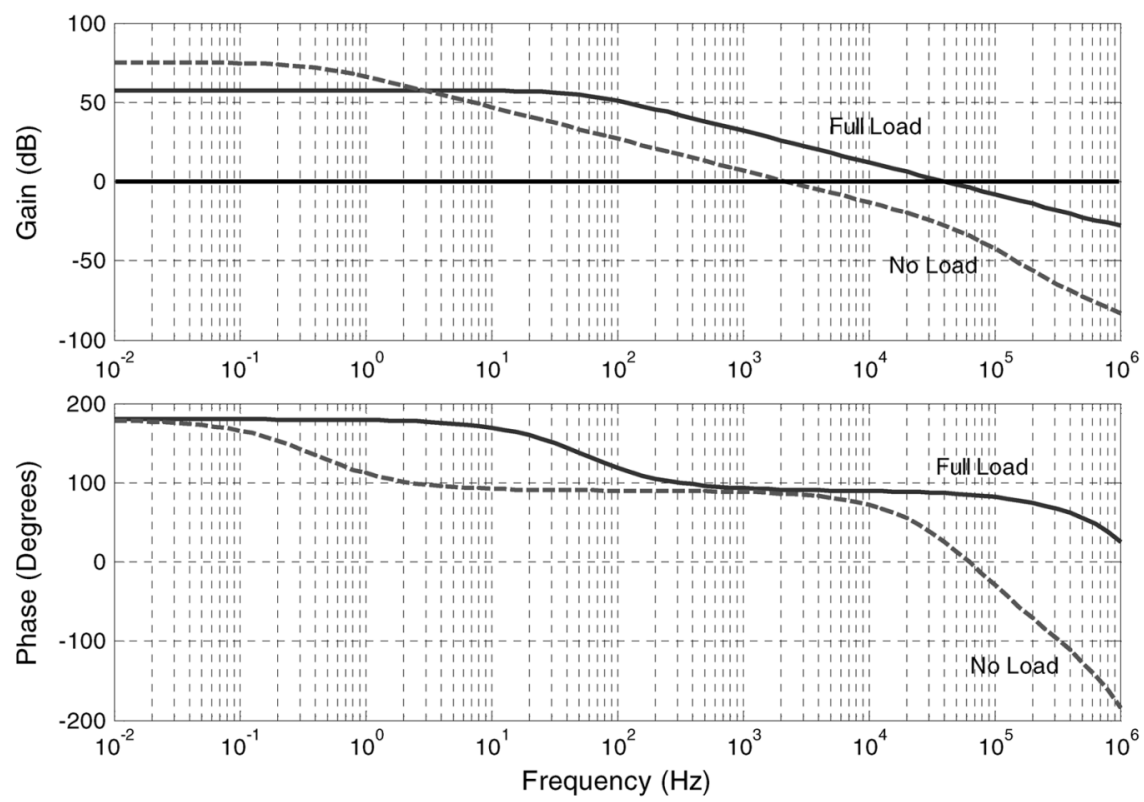

Fig. 6. Simulated loop-gain transfer function of the proposed LDO with a $1 \mu \mathrm{F}$ output capacitor.

Similar to the derivation in (10) and (11), the loop-gain transfer function of the proposed LDO using current-buffer compensation is actually a second-order system under the full range of the load current and $T(s)$ from (6) can then be simplified as

$$
\begin{aligned}
T(s) & \approx \frac{-B g_{m 1} g_{m p} r_{o 1} R_{o e q}}{1+s R_{o e q}\left(C_{L}+g_{m p} r_{o 1} C_{c}\right)+s^{2} C_{1} C_{L} r_{o 1} R_{o e q}} \\
& \approx \frac{-B g_{m 1} g_{m p} r_{o 1} R_{o e q}}{\left[1+s R_{o e q}\left(C_{L}+g_{m p} r_{o 1} C_{c}\right)\right]\left(1+s \frac{C_{1} C_{L} r_{o 1}}{\left(C_{L}+g_{m p} r_{o 1} C_{c}\right)}\right)} \\
& =\frac{-B g_{m 1} g_{m p} r_{o 1} R_{o e q}}{\left(1+p_{-3 \mathrm{~dB}}\right)\left(1+p_{\mathrm{nd}}\right)} .
\end{aligned}
$$

Equation (12) can be used to find out the worst-case stability of the LDO under different load currents such that the value of the compensation capacitor $C_{c}$ can be designed to ensure the stability of the LDO in the entire load current range. The worst-case stability occurs at the minimum phase margin of the second-order system. The phase margin (PM) of a compensated two-pole system is given by

$$
\mathrm{PM}=90^{\circ}-\arctan \frac{\omega_{u}}{p_{\text {nd }}}
$$

where $\omega_{u}$ is the unity-gain frequency. The larger the ratio $p_{\text {nd }} / \omega_{u}$ is, the better the PM of the system can achieve. As a result, $F_{\mathrm{PM}}$ is defined to evaluate the separation between $p_{\text {nd }}$ and $\omega_{u}$ under different load currents, which is given as

$$
F_{\mathrm{PM}}=\frac{p_{\mathrm{nd}}}{\omega_{u}}=\frac{\omega_{p_{\mathrm{nd}}}}{A_{\mathrm{DC}} \cdot p_{-3 \mathrm{~dB}}}
$$

where $A_{\mathrm{DC}}$ is the $\mathrm{DC}$ loop-gain magnitude given by $B g_{m 1} g_{m p} r_{o 1} R_{o e q}$. By substituting $p_{\mathrm{nd}}, p_{-3 \mathrm{~dB}}$ and $A_{\mathrm{DC}}$ from (12) into (14) and setting $B=1[B=1$ corresponds to the worst-case feedback factor for stability as it maximizes the denominator of (14)], $F_{\mathrm{PM}}$ can be rewritten as

$$
F_{\mathrm{PM}}=\frac{\left(C_{L}+g_{m p} \cdot r_{o 1} C_{c}\right)^{2}}{g_{m p} \cdot\left(g_{m 1} C_{1} C_{L} r_{o 1}^{2}\right)}
$$

If we differentiate $F_{\mathrm{PM}}$ with respect to $g_{m p}$ and set the derivative to zero, the minimum value of $F_{\mathrm{PM}}$ can be found, at which the minimum phase margin occurs. The resulting minimum $F_{\mathrm{PM} \text { min }}$ is given by

$$
F_{\mathrm{PM}, \min }=\frac{4}{g_{m 1} r_{o 1}} \cdot \frac{C_{c}}{C_{1}} .
$$

By substituting (16) into (13), the minimum phase margin $\mathrm{PM}_{\min }$ is given as

$$
\mathrm{PM}_{\text {min }}=90^{\circ}-\arctan \left(\frac{g_{m 1} r_{o 1}}{4} \cdot \frac{C_{1}}{C_{c}}\right)
$$

and from (17), the $C_{c}$ can be designed as

$$
C_{c}=\frac{g_{m 1} r_{o 1}}{4 \cdot \tan \left(90^{\circ}-\mathrm{PM}_{\min }\right)} \cdot C_{1} .
$$

From (18), $C_{c}$ is proportional to small parasitic capacitance $C_{1}$. Accordingly, the impedance-attenuated buffer which results in a small $C_{1}$ reduces the required value of $C_{c}$. In our design, a $C_{c}$ of $10 \mathrm{pF}$ is sufficient for the LDO to be stable under the full range of the load current. This capacitance can be easily integrated in CMOS technologies.

The simulated loop gain transfer functions of the proposed LDO with $C_{L}=1 \mu \mathrm{F}$ under no-load and full-load conditions are shown in Fig. 6. Simulated results validate the existence of only a single pole within the unity-gain frequency and a good phase margin of approximately $90^{\circ}$ is achieved under both conditions. In addition, Fig. 7 shows the simulated phase margin of the loop-gain transfer function under different load currents. The minimum phase margin is always larger than $65^{\circ}$ for the entire range of the load current. This simulation result further verifies that a small $C_{c}$ of $10 \mathrm{pF}$ is sufficient to ensure unconditionally stability under the full range of the load current. 


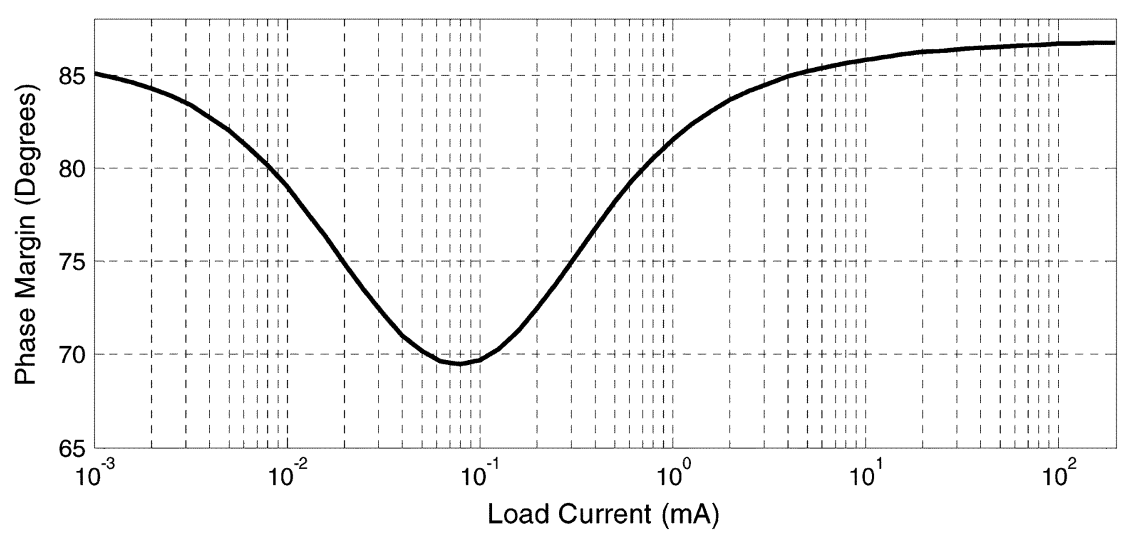

Fig. 7. Phase margin of the proposed LDO loop-gain transfer function under different load currents with a $1-\mu \mathrm{F}$ output capacitor.

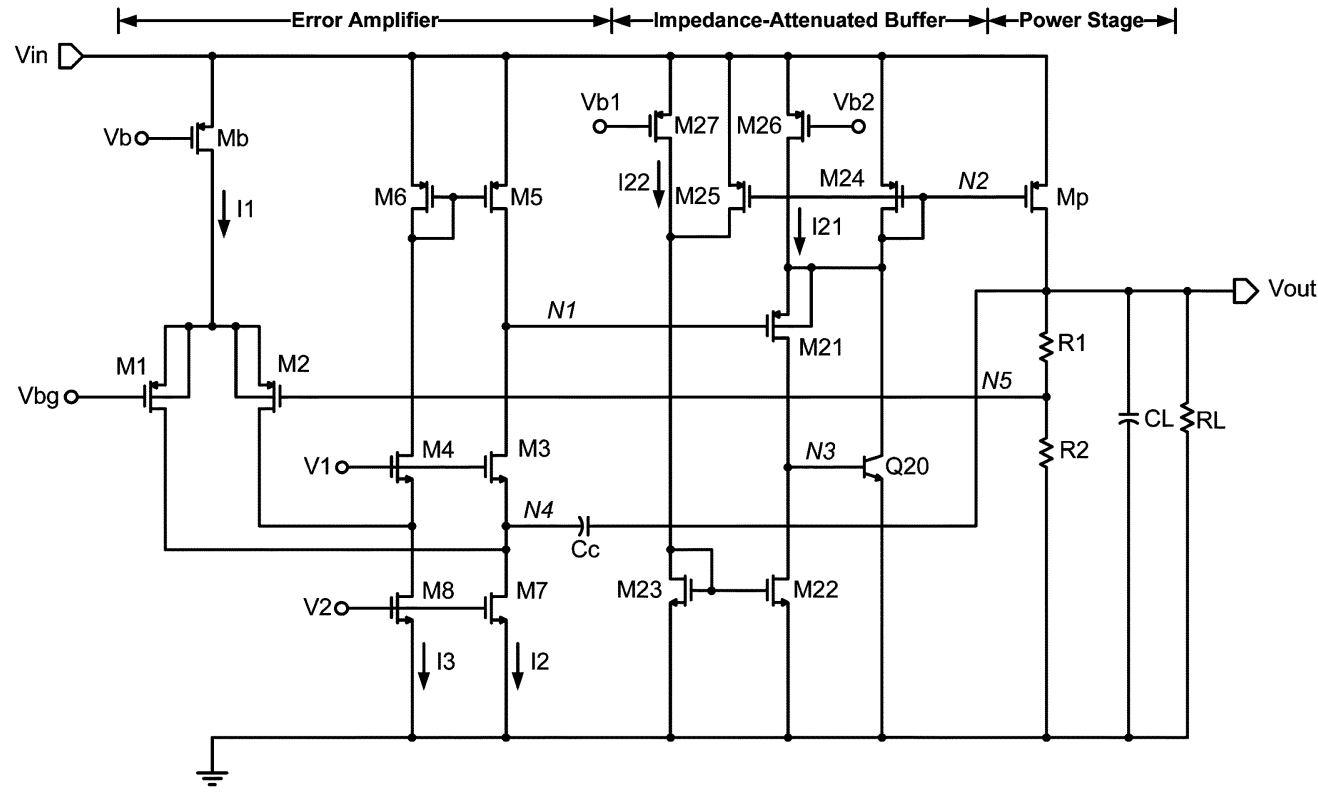

Fig. 8. Schematic of the proposed LDO.

\section{B. Circuit Design}

The schematic of the proposed LDO is shown in Fig. 8 [16]. A $1-\mathrm{V}$ bandgap voltage reference is integrated together with the LDO to provide the reference voltage $V_{b g}$ to the input of the error amplifier. In the single-stage folded-cascode error amplifier, a simple diode-connected active load using transistors $M_{5}$ and $M_{6}$ is adopted. The simple diode-connected active load results in a smaller output resistance of the error amplifier $r_{o 1}$ as compared to a cascode load, which helps reduce the value of compensation capacitor $C_{c}$ to achieve a particular phase margin according to (18). In the proposed LDO, the gain of the error amplifier is equal to $g_{m 1} r_{o 1}$ and can always be greater than $50 \mathrm{~dB}$ under the entire load current range.

Moreover, the use of a pMOS transistor to implement the source-follower in the proposed buffer is extremely critical to minimize the quiescent current at no-load condition. Under no-load condition, the pass device $M_{p}$ is almost turned off, as it only provides current for the feedback resistors $R_{1}$ and $R_{2}$. The current is equal to $V_{\text {out }} /\left(R_{1}+R_{2}\right)$, and the gate-source voltage $V_{s g}$ required for $M_{p}$ to minimize the amount of current can be around $100 \mathrm{mV}$. In order to allow the error amplifier to maintain its gain, the DC voltage level at the output of the error amplifier needs to be at least an overdrive voltage $V_{o v} \approx$ $200 \mathrm{mV}$ below $V_{\mathrm{in}}$. If the buffer stage has no level shifting toward the input supply, $V_{s g}$ of the pass device would need to be increased under no-load condition in order to allow the DC voltage at $N_{1}$ to be within the output swing of the error amplifier. As a result, both $R_{1}$ and $R_{2}$ need to be reduced to accommodate the required increase in $V_{s g}$ of $M_{p}$, which results in an undesirable increase in the quiescent current in the LDO under no-load condition.

\section{EXPERIMENTAL RESULTS AND DISCUSSIONS}

In order to verify the proposed buffer impedance attenuation technique, the LDO shown in Fig. 8 was fabricated in a $0.35-\mu \mathrm{m}$ twin-well $7-\mathrm{V}$ CMOS process. Fig. 9 shows the die micrograph of the proposed LDO, which was fabricated as part of an integrated power IC. The effective die area of the LDO is $0.264 \mathrm{~mm}^{2}$. It should be noted that the output voltage of the LDO is bonded with only a single bonding wire from the pad to the pin in order to minimize the cost.

The input voltage range of the LDO is designed from $2.0 \mathrm{~V}$ to $5.5 \mathrm{~V}$ for portable applications using lithium-ion batteries. This 


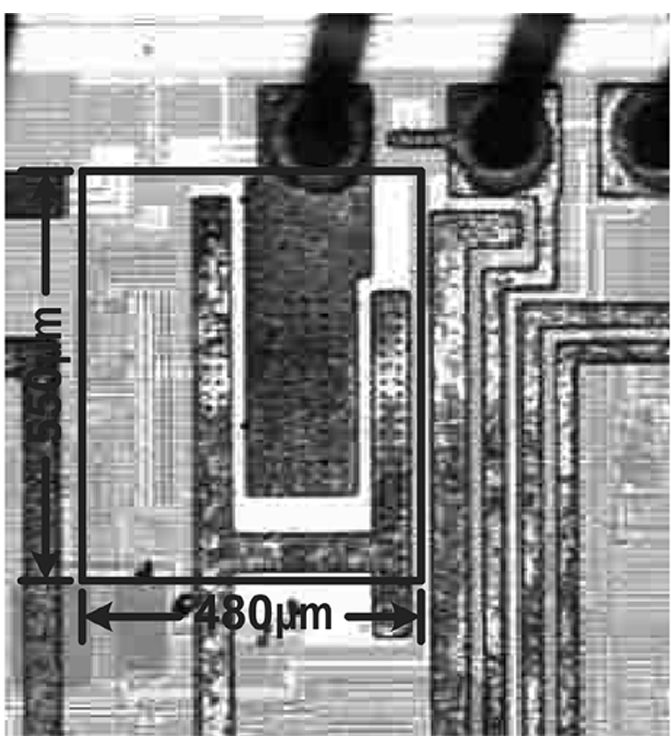

Fig. 9. Micrograph of the proposed LDO.

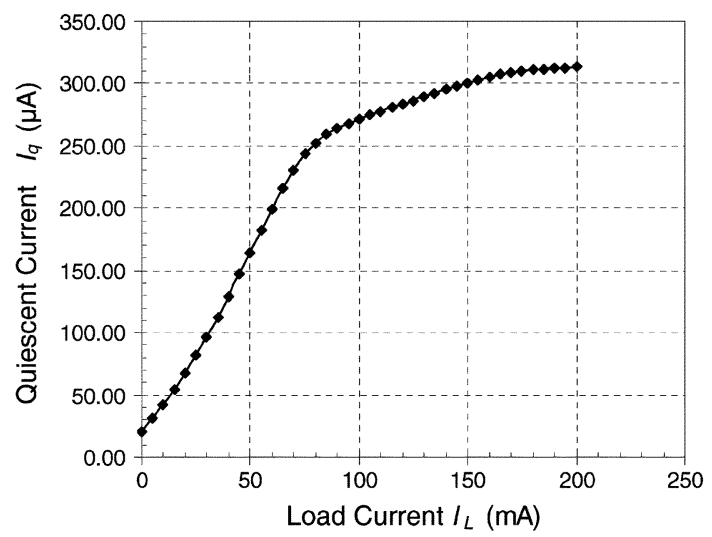

Fig. 10. Measured quiescent current under different load currents.

covers the widest lithium-ion battery variation, which ranges from $2.5 \mathrm{~V}$ to $5.5 \mathrm{~V}$. The output voltage of the LDO has programmable steps with a step size of $50 \mathrm{mV}$ and the minimum output voltage is $1.8 \mathrm{~V}$. When $V_{\text {out }}=1.8 \mathrm{~V}$, the LDO can deliver up to $200 \mathrm{~mA}$ with a dropout voltage of $0.2 \mathrm{~V}$. The output capacitor range is from $1 \mu \mathrm{F}$ to $100 \mu \mathrm{F}$. Fig. 10 shows the measured quiescent current $I_{q}$ under different load currents $I_{L}$. The figure indicates that the quiescent current increases with $I_{L}$, which validates the dynamic increase in current realized by transistors $M_{24}$ and $M_{25}$ to lower the output resistance of the intermediate buffer stage. In addition, from Fig. 10, the LDO consumes small quiescent current of $20 \mu \mathrm{A}$ under no-load condition, while the quiescent current of $320 \mu \mathrm{A}$ is dissipated at full-load condition. It should be noted that the maximum quiescent current seen over multiple samples at full load is $340 \mu \mathrm{A}$, which corresponds to the worst-case current efficiency of $99.8 \%$ in the proposed LDO. Table I provides the detailed measurement results.

Fig. 11(a) shows the transient response of the proposed LDO when the load current is pulsating between $1 \mathrm{~mA}$ and $200 \mathrm{~mA}$ with pulse rise and fall time of $100 \mathrm{~ns}$. With the use of a $1-\mu \mathrm{F}$ output ceramic capacitor, the maximum output-voltage variation is less than $40 \mathrm{mV}$ when $V_{\text {out }}$ is at $1.8 \mathrm{~V}$, which includes
TABLE I

PERFORMANCE SUMMARY OF PROPOSED LDO

\begin{tabular}{c|c}
\hline Full Load Current & $200 \mathrm{~mA}$ \\
\hline Output Capacitor & $1 \mu \mathrm{F}$ \\
\hline$I_{q}$ (no-load) & $20 \mu \mathrm{A}$ \\
\hline$I_{q}$ (full-load) & $340 \mu \mathrm{A}$ \\
\hline Dropout Voltage $V_{\mathrm{do}}$ & $0.2 \mathrm{~V}$ \\
\hline DC Load Regulation & $34 \mathrm{mV}=170 \mu \mathrm{V} / \mathrm{mA}$ \\
0 - 200mA & $($ single bondwire) \\
\hline DC Line Regulation & $2 \mathrm{mV} / \mathrm{V}\left(\Delta V_{\mathrm{in}}=3.5 \mathrm{~V}\right)$ \\
\hline PSRR (0-20kHz) & $>45 \mathrm{~dB}$ \\
\hline Transient Response & Undershoot $<1.1 \%$ \\
0 - 200mA Step & $C_{L}=1 \mu \mathrm{F}$ \\
\hline Transient Response & Undershoot $<0.9 \%$ \\
1 - 200mA Step & $C_{L}=1 \mu \mathrm{F}$ \\
\hline Die Area & $0.264 \mathrm{~mm}^{2}$ \\
\hline Technology & $0.35 \mu \mathrm{m} \mathrm{CMOS}$ \\
\hline
\end{tabular}

output undershoots, overshoots and variations due to load regulation. This $40-\mathrm{mV}$ total output variation corresponds to slightly more than $2 \%$ of $V_{\text {out }}$. The total output undershoots and overshoots are less than $15 \mathrm{mV}$ above and below the steady-state $V_{\text {out }}$, which amounts to less than $1 \%$ of the output voltage. With the same output capacitor, when the LDO is tested with full-load steps pulsating between 0 and $200 \mathrm{~mA}$, Fig. 11(b) shows that the maximum output-voltage variation is $54 \mathrm{mV}$, which corresponds to $3 \%$ of $V_{\text {out }}$. The transient undershoot of $20 \mathrm{mV}$ only accounts for $1.1 \%$ of $V_{\text {out }}$. Fig. 12 shows the measured load transient response when output voltage of the LDO is changed to $3.15 \mathrm{~V}$. In this case, the maximum output voltage variation is $65 \mathrm{mV}$, which is $2 \%$ of $V_{\text {out }}=3.15 \mathrm{~V}$. The transient undershoot for this condition is $25 \mathrm{mV}$, which only corresponds to $0.8 \%$ of $V_{\text {out }}$.

Based on measured results shown in Figs. 11(a) and (b) and 12 , transient overshoots and undershoots only occupy a small portion of the total output-voltage variation under massive load-step changes even when a small output capacitor of $1 \mu \mathrm{F}$ is used. This is because both the proposed impedance-attenuated buffer and current-buffer compensation allow the LDO to achieve stability without relying on any low-frequency ESR zero. Moreover, the well-behaved transient settling behavior of the output voltage further justifies large phase margin achieved in the LDO by using the proposed techniques.

As mentioned before, the LDO output is bonded with a single bonding wire to the pin in our design and the resistance of the bondwire varies from $50 \mathrm{~m} \Omega$ to $100 \mathrm{~m} \Omega$. The IR drop across the bondwire can then be as high as $20 \mathrm{mV}$ when the load current is $200 \mathrm{~mA}$. This accounts for almost $60 \%$ of the total DC load regulation error of $34 \mathrm{mV}$ in Fig. 11(b). This error can be reduced if multiple bonding wires are used to decrease the bondwire resistance. As a result, both the DC load regulation and the total output-voltage variation during transient can be minimized.

The measured line transient response of the proposed LDO is shown in Fig. 13 in order to evaluate the effect of battery voltage 


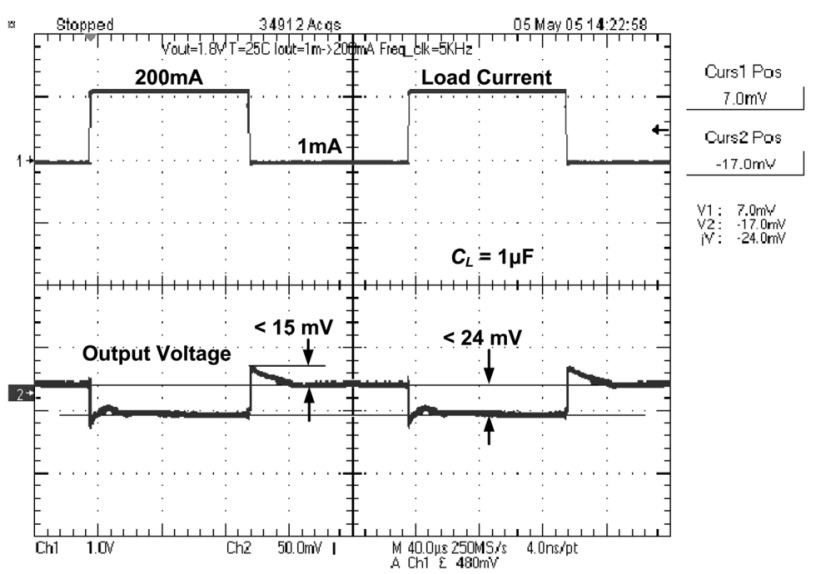

(a)

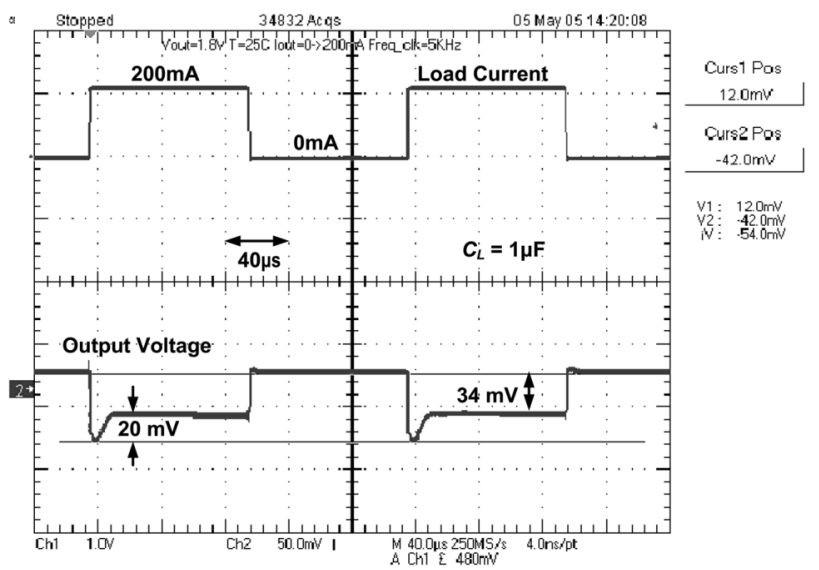

(b)

Fig. 11. Measured load transient response of the proposed LDO for a load step pulsating (a) between $1 \mathrm{~mA}$ and $200 \mathrm{~mA}$ and (b) between 0 and $200 \mathrm{~mA}$ with rise and fall time of $100 \mathrm{~ns}$ and $V_{\text {out }}=1.8 \mathrm{~V}$.

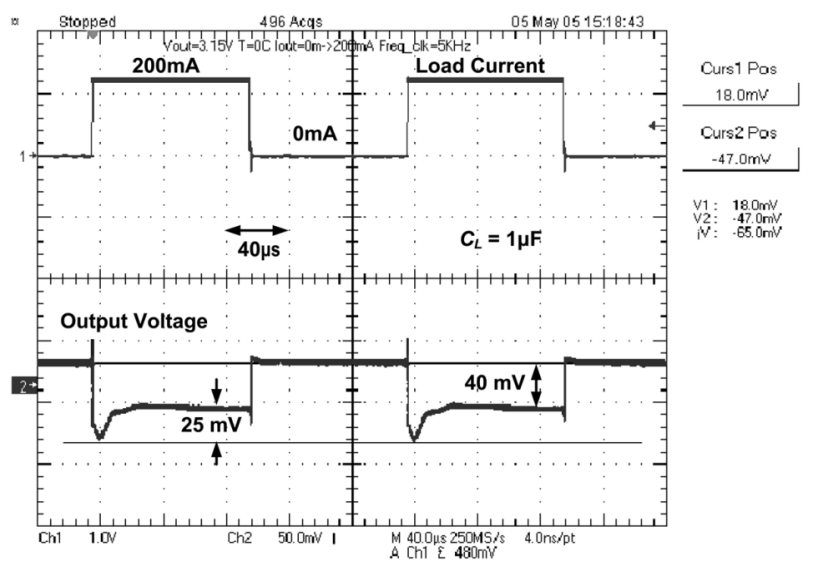

Fig. 12. Measured load transient response of the proposed LDO for a load step pulsating between 0 and $200 \mathrm{~mA}$ with rise and fall time of $100 \mathrm{~ns}$ and $V_{\text {out }}=$ $3.15 \mathrm{~V}$.

changes on the LDO output voltage. When the change in the input supply of the LDO is $1 \mathrm{~V}$ with rise and fall time of $40 \mu \mathrm{s}$, the output voltage changes by less than $3 \mathrm{mV}$ at $I_{L}=1 \mathrm{~mA}$.

In order to provide a clearer picture of the performance improvement in the proposed LDO resulting from the impedance-attenuated buffer and current-buffer compensation, a comparison of some reported LDOs is given in Table II.

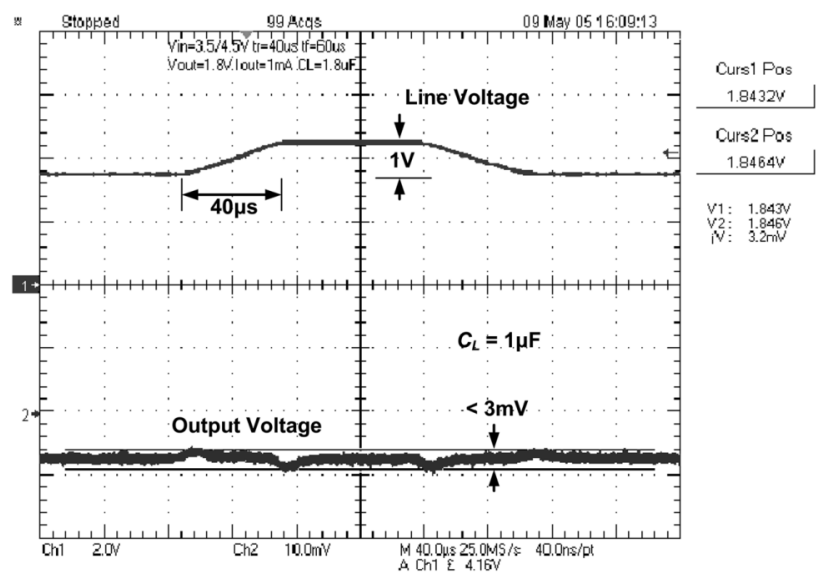

Fig. 13. Measured line transient response of the proposed LDO for a line step change of $1 \mathrm{~V}$.

A figure of merit FOM $=T_{r}\left(I_{q} / I_{L, \max }\right)$ [17] is adopted to compare the transient response of different LDOs by evaluating the tradeoffs between the response time $T_{r}$, the quiescent current $I_{q}$ and the maximum load current $I_{L \text {, max }} . T_{r}$ of the LDO is given by $T_{r}=\left(C_{L} \Delta V_{\text {out }}\right) / I_{L, \max }$, where $\Delta V_{\text {out }}$ is the maximum transient output-voltage variation (the peak-to-peak output-voltage change due to overshoots, undershoots and load-regulation error during load transient). The smaller the FOM, the better the transient response the LDO achieves. From Table II, the proposed LDO achieves the smallest FOM compared with other reported LDOs. As the proposed LDO is designed for portable applications, the FOM achieved by the proposed LDO is much smaller than other reported LDOs for the same applications [1], [3]-[5] by at least one order of magnitude. It should be noted that the LDO for embedded microprocessor applications [17] dissipates much larger quiescent current in full-load condition, as compared to other LDOs for portable applications. Hence, the current efficiency of the LDO in [17] is much lower. Current efficiency above $99 \%$ is critical if the LDO is designed for portable applications as the current efficiency determines the battery lifetime.

\section{CONCLUSION}

In this paper, a low-dropout regulator based on the proposed buffer-impedance attenuation (BIA) technique with dynamically-biased shunt feedback has been presented. The BIA technique enables the realization of an intermediate pass-device driving stage with minimal capacitive loading to the preceding error amplifier stage, and minimal output resistance driving the pass device. The parasitic pole at the gate of the pass device can be pushed to sufficiently high frequencies under different load currents, while the LDO only dissipates low quiescent current. In addition to the BIA technique, current-buffer compensation allows the LDO to achieve only a single pole within the unity-gain bandwidth of the regulation loop and a good phase margin with a small compensation capacitor. The LDO is thus stable under the full range of the load current without any requirement for a low-frequency zero. Good transient response of the LDO with small undershoots and overshoots is achieved even if an inexpensive low-value 
TABLE II

PERFormance COMPARISON WiTh PREVIOUSLY REPORTED LDOS

\begin{tabular}{c|c|c|c|c|c|c}
\hline & $\begin{array}{c}1998 \\
{[1]}\end{array}$ & $\begin{array}{c}2000 \\
{[3]}\end{array}$ & $\begin{array}{c}2003 \\
{[4]}\end{array}$ & $\begin{array}{c}2004 \\
{[5]}\end{array}$ & $\begin{array}{c}2005 \\
{[17]}\end{array}$ & $\begin{array}{c}\text { This } \\
\text { Work }\end{array}$ \\
\hline \hline Technology $(\mu \mathrm{m})$ & 2.0 & 1.0 & 0.6 & 0.5 & 0.09 & $\mathbf{0 . 3 5}$ \\
\hline$V_{\text {do }}(\mathrm{V})$ & 0.3 & N.A. & 0.2 & N.A. & 0.3 & $\mathbf{0 . 2}$ \\
\hline$I_{L, \max }(\mathrm{mA})$ & 50 & 200 & 100 & 160 & 100 & $\mathbf{2 0 0}$ \\
\hline$I_{q}(\mathrm{~mA})$ & 0.023 & 0.03 & 0.038 & 0.025 & 6 & $\mathbf{0 . 0 2}$ \\
\hline Current Efficiency at $I_{L, \max }(\%)$ & 99.5 & N.A. & N.A. & N.A. & 94.3 & $\mathbf{9 9 . 8}$ \\
\hline$\Delta V_{\text {out }}(\mathrm{mV})$ & 19 & 220 & 130 & 200 & 90 & $\mathbf{5 4}$ \\
\hline$T_{r}(\mu \mathrm{s})$ & 1.8 & 1.1 & 2 & 2.75 & 0.00054 & $\mathbf{0 . 2 7}$ \\
\hline$C_{L}(\mu \mathrm{F})$ & 4.7 & 1 & 10 & 2.2 & 0.00060 & $\mathbf{1}$ \\
\hline ESR Zero Required & No & Yes & Yes & No & No & No \\
\hline FOM $(\mathrm{ns})$ & 8.2 & 0.165 & 4.9 & 0.43 & 0.032 & $\mathbf{0 . 0 2 7}$ \\
\hline
\end{tabular}

output capacitor is used. The LDO is able to achieve low dropout voltage of $200 \mathrm{mV}$ and deliver 200-mA load current for portable power management applications with lithium-ion batteries. This work is verified on silicon and comparisons with the previous reported work on low-dropout regulators are also presented.

\section{ACKNOWLEDGMENT}

The authors would like to thank D. Aksin, M. Martins, and G. Balachandran for their useful input throughout the implementation of this work.

\section{REFERENCES}

[1] G. A. Rincon-Mora and P. E. Allen, "A low-voltage, low quiescent current, low drop-out regulator," IEEE J. Solid-State Circuits, vol. 33, no. 1, pp. 36-44, Jan. 1998.

[2] G. A. Rincon-Mora and P. E. Allen, "Optimized frequency-shaping circuit toplogies for LDO's," IEEE Trans. Circuits Syst. II, Analog Digit. Signal Process., vol. 45, no. 6, pp. 703-708, Jun. 1998.

[3] G. A. Rincon-Mora, "Active capacitor multiplier in Miller-compensated circuits," IEEE J. Solid-State Circuits, vol. 35, no. 1, pp. 26-32, Jan. 2000.

[4] K. N. Leung and P. K. T. Mok, "A capacitor-free CMOS low-dropout regulator with damping-factor-control-frequency compensation," IEEE J. Solid-State Circuits, vol. 38, no. 10, pp. 1691-1702, Oct. 2003.

[5] C. K. Chava and J. Silva-Martinez, "A frequency compensation scheme for LDO voltage regulators," IEEE Trans. Circuits Syst. I, vol. 51, no. 6, pp. 1041-1050, Jun. 2004.

[6] B. Y. Kamath, R. G. Meyer, and P. R. Gray, "Relationship between frequency response and settling time of operational amplifier," IEEE J. Solid-State Circuits, vol. SC-9, pp. 347-352, Dec. 1974.

[7] C. T. Chuang, "Analysis of the settling behavior of an operational amplifier," IEEE J. Solid-State Circuits, vol. SC-17, pp. 74-80, Feb. 1982.

[8] P. R. Gray, P. J. Hurst, S. H. Lewis, and R. G. Meyer, Analysis and Design of Analog Integrated Circuits, 4th ed. New York: Wiley, 2001.

[9] B. K. Ahuja, "An improved frequency compensation technique for CMOS operational amplifiers," IEEE J. Solid-State Circuits, vol. SC-18, no. 6, pp. 629-633, Dec. 1983.

[10] R. Reay and G. Kovacs, "An unconditionally stable two-stage CMOS amplifier," IEEE J. Solid-State Circuits, vol. 30, no. 5, pp. 591-594, May 1995.
[11] G. Palmisano and G. Palumbo, "A compensation strategy for two-stage CMOS opamps based on current buffer," IEEE Trans. Circuits Syst. I, Fundam. Theory Applicat., vol. 44, no. 3, pp. 257-262, Mar. 1997.

[12] B. Razavi, Design of Analog CMOS Integrated Circuits. New York: McGraw-Hill, Inc., 2001

[13] D. B. Ribner and M. A. Copeland, "Design techniques for cascoded CMOS op amps with improved PSRR and common-mode input range," IEEE J. Solid-State Circuits, vol. SC-19, no. 12, pp. 919-925, Dec. 1984.

[14] R. Hogervorst, J. P. Tero, and J. H. Huijsing, "A compact power-efficient $3 \mathrm{~V}$ CMOS rail-to-rail input/output operational amplifier for VLSI cell libraries," IEEE J. Solid-State Circuits, vol. 29, no. 12, pp. 1505-1513, Dec. 1994.

[15] H. Lee, K. N. Leung, and P. K. T. Mok, "Low-voltage analog circuit techniques using bias-current reutilization, self-biasing and signal superposition," in Proc. IEEE Int. Conf. Electron Devices and Solid-State Circuits, 2005, pp. 533-536.

[16] M. Al-Shyoukh, R. A. Perez, and H. Lee, "A transient-enhanced $20-\mu$ A-quiescent $200 \mathrm{~mA}$-load low-dropout regulator with buffer impedance attenuation," in Proc. IEEE Custom Integrated Circuits Conf. (CICC), 2006, pp. 615-618.

[17] P. Hazucha, T. Karnik, B. A. Bloechel, C. Parsons, D. Finan, and S. Borkar, "Area-efficient linear regulator with ultra-fast load regulation," IEEE J. Solid-State Circuits, vol. 40, no. 4, pp. 933-940, Apr. 2005.

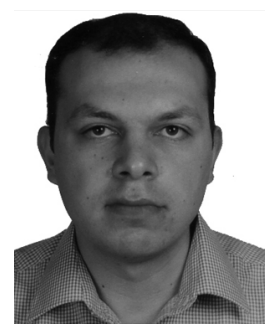

Mohammad Al-Shyoukh received the B.Sc. degree from the electrical and computer engineering Department, Jordan University of Science and Technology, Irbid, Jordan, in 1997, and the M.Sc. degree from the Electrical and Computer Engineering Department, The Ohio State University, Columbus, OH, in 1999 $\mathrm{He}$ is currently working toward the Ph.D. degree in electrical engineering at the University of Texas at Dallas.

In 2000, he joined Tezzaron Semiconductor as an analog and mixed-signal IC designer where he worked on memory and stacked memory development, ultra-low-power linear voltage regulators, opamps, and SAR converters. Since late 2002, he has been with Texas Instruments Inc., Dallas, TX, working in the Mixed Signal Automotive and later the Wireless Terminals organizations. He has been involved in the development of wide range of power ICs for automotive and handheld applications. His research interests include linear and power IC design, and ultra-low-power data converters. 


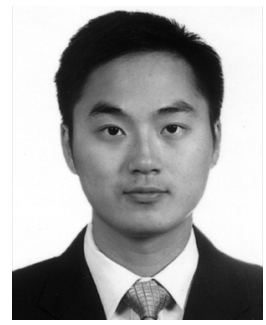

Hoi Lee (S'00-M'05) received the B.Eng. (First Class Honors), M.Phil., and Ph.D. degrees in electrical and electronic engineering from the Hong Kong University of Science and Technology, Hong Kong, in 1998, 2000, and 2004, respectively.

In January 2005, he joined the Department of Electrical Engineering, University of Texas at Dallas, Richardson, TX, as an Assistant Professor. His current research interests include power management integrated circuits, integrated systems for cochlear and neural prostheses and low-voltage low-power analog and mixed-signal circuit techniques.

Dr. Lee was the recipient of the Best Student Paper Award at the 2002 IEEE Custom Integrated Circuits Conference. He serves as a member of the Technical Program Committee of IEEE Asian Solid-State Circuits Conference (A-SSCC) and the Analog Signal Processing Technical Committee of the IEEE Circuits and Systems Society.

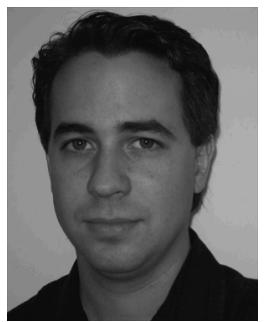

Raul Perez (M'06) received the B.S.E.E. degree from the University of Puerto Rico, Mayaguez Campus, and the M.S.E.E. degree from the University of Texas at Dallas in 2000 and 2005, respectively. In 2000, he joined the Wireless Terminals group at Texas Instruments Inc., Dallas, TX, where he worked on power and linear IC design. His design expertise includes low-dropout regulators (LDOs), low-power, low voltage and low noise analog design, single-ended and fully-differential amplifier architectures, and voltage and current references references in CMOS, BiCMOS and BCD technologies. He developed several products for the major wireless phone vendors, as well as the catalog and consumer electronics markets. In 2006, he moved to Fyrestorm Inc., Sunnyvale, $\mathrm{CA}$, where he is currently Senior Analog and Power IC Designer and team lead. His technical interests include DC/DC converters, Class-D amplifiers and silicon carbide ICs for hybrid electric vehicle applications. He currently holds four U.S. patents with three more pending, all in linear and power IC design. 